\title{
Maternal Exposure to Local, Source-specific Ambient Air Pollution During Pregnancy and Autism in Children: a Cohort Study From Southern Sweden
}

\section{Erin Flanagan ( $\nabla$ erin.flanagan@med.lu.se )}

Division of Occupational and Environmental Medicine, Department of Laboratory Medicine, Faculty of Medicine, Lund University

\section{Ebba Malmqvist}

Division of Occupational and Environmental Medicine, Department of Laboratory Medicine, Faculty of Medicine, Lund University

\section{Ralf Rittner}

Division of Occupational and Environmental Medicine, Department of Laboratory Medicine, Faculty of Medicine, Lund University

\section{Peik Gustafsson}

Child and Adolescent Psychiatry, Department of Clinical Sciences, Faculty of Medicine, Lund University

Karin Källén

Division of Occupational and Environmental Medicine, Department of Laboratory Medicine, Faculty of Medicine, Lund University

\section{Anna Oudin}

Division of Occupational and Environmental Medicine, Department of Laboratory Medicine, Faculty of Medicine, Lund University

\section{Research Article}

Keywords: ambient air pollution, fine particulate matter, local air pollution, source-specific exposure, traffic-related air pollution, residential burning, wood smoke, autism, autism spectrum disorders

Posted Date: November 1st, 2021

DOI: https://doi.org/10.21203/rs.3.rs-1027824/v1

License: (c) (i) This work is licensed under a Creative Commons Attribution 4.0 International License. Read Full License 


\section{Abstract}

Evidence of air pollution exposure, namely, ambient particulate matter (PM), during pregnancy and an increased risk of autism spectrum disorders (ASD) in children is growing. Which PM sources contribute to this association is currently unknown, however. The aim of the present study was to investigate local source-specific ambient PM exposure and its association with ASD. A cohort of 48,571 singleton births from 1999 to 2009 in Scania, Sweden, was combined with data on locally emitted PM with an aerodynamic diameter $<2.5 \mu \mathrm{m}\left(\mathrm{PM}_{2.5}\right)$. A flat, two-dimensional dispersion model was used to model $\mathrm{PM}_{2.5}$ exposure (all-source $\mathrm{PM}_{2.5}$, tailpipe exhaust, vehicle wear-and-tear, and small-scale residential heating- mainly wood burning) at the residential address. Associations were analyzed using binary logistic regression in partially and fully adjusted models. Clear associations were observed between $\mathrm{PM}_{2.5}$ and $\mathrm{ASD}$, with statistical significance for all investigated sources in the partially adjusted model. In the fully adjusted model, only all-source $\mathrm{PM}_{2.5}$ was statistically significant. The results add to existing evidence that exposure to air pollution during pregnancy may be associated with an increased risk for ASD among children. Further, these findings suggest that locally emitted tailpipe exhaust, vehicle wearand-tear, and small-scale residential heating all contribute to this association.

\section{Introduction}

Air pollution is a major cause of morbidity and mortality worldwide ${ }^{1}$. For example, particulate matter (PM) with an aerodynamic diameter less than $2.5 \mu \mathrm{m}\left(\mathrm{PM}_{2.5}\right)$ derived from fossil fuels alone has recently been estimated to contribute to 10.2 million premature deaths around the world each year, which corresponds to one in five deaths ${ }^{2}$. The relationship between traffic-related air pollution, often tailpipe emissions stemming from diesel fuel and petrol combustion, and adverse health effects are well established ${ }^{3,4}$. Aside from traffic and fossil fuels, wood burning also produces PM as well as other toxic compounds ${ }^{5-7}$, yet little is known about the health effects of ambient wood smoke. This is generally because prior research on the health impacts of $\mathrm{PM}_{2.5}$ has typically been based on exposure from aggregate particle mass rather than specific sources. However, there is evolving evidence indicating that health effects may vary in type and degree depending on the source of $\mathrm{PM}_{2.5}$ exposure ${ }^{8}$. Source-specific epidemiological studies are still scarce, though, as such separation requires high quality exposure data that has not been available previously. Because air pollution is far from static but varies substantially in time and space, advanced exposure assessment can better capture local exposures' unique composition. Indeed, an advantage of using such methods is the ability to distinguish the diverse health effects of distinct, specified sources, rather than from the total concentration. For instance, associations between vehicle exhaust particles and low birth weight have been observed in a Swedish study using advanced sourcespecific exposure data ${ }^{9}$. Associations between ambient wood smoke, and their related markers, and both dementia and childhood asthma ${ }^{10,11}$ have also been found in other studies using advanced exposure assessment data. 
While all people are susceptible to the adverse health effects of air pollution, pregnant women and their developing fetuses may be particularly vulnerable. For instance, exposure to various air pollutants has been associated with a range of adverse pregnancy ${ }^{12}$ and birth outcomes ${ }^{13}$. An experimental cell study reported that intracellular accumulation of PM may contribute to the placental dysfunction associated with unfavorable pregnancy outcomes, such as preeclampsia and intrauterine growth restriction, through their direct and indirect effects on trophoblast protein secretion, hormone regulation, inflammatory response, and mitochondrial interference ${ }^{14}$. Considering source-apportionment studies, associations have been identified between $\mathrm{PM}_{10}$ from brake dust and combined traffic-related sources with preeclampsia ${ }^{15}$. Preterm birth was found to be associated with total $\mathrm{PM}_{2.5}$ and $\mathrm{PM}_{2.5}$ derived from traffic, oil combustion, as well as secondary sulfates and organics (derived from a reaction of ammonia from livestock and sulfuric acid from fuel ${ }^{16}$. Moreover, $\mathrm{PM}_{2.5}$ total mass, secondary ammonium sulfate, secondary ammonium nitrate (derived from a reaction of ammonia and nitric acid from vehicular sources), and resuspended soil (i.e., road dust) were associated with low birth weight ${ }^{17}$. A further source-apportionment study found associations between stillbirth and vehicular emissions, re-suspended soil, and secondary ammonium sulfate ${ }^{18}$. Beyond these immediate complications, exposure to air pollution during pregnancy can lead to adverse health outcomes later in the child's life. Indeed, the in-utero period can affect immunologic, respiratory, gastrointestinal, and central nervous system development; delay motor, hearing, visual, cognitive, behavioral, and social-emotional function; and impact their overall health and wellbeing ${ }^{19}$. This has been termed "fetal programming" 20 .

With this, an additional health outcome has been considered in connection to air pollution exposure during pregnancy and in early life: autism spectrum disorders (ASD). Evidence of this association has been developing, with a 2016 systematic review and meta-analysis supporting the association but emphasizing cautious interpretation due to the relatively small sample of available studies ${ }^{21}$. A 2019 systematic review and meta-analysis including both adults and children has demonstrated clearer associations, naming ASD among other neurological and developmental disorders associated with $\mathrm{PM}_{2.5}$ exposure $^{22}$. Two, more current, systematic reviews and meta-analyses focusing on children only and prenatal exposure specifically identified the strongest evidence for $\mathrm{PM}_{2.5}$ exposure and $\mathrm{ASD}^{23,24}$, with one reporting each $5 \mu \mathrm{g} / \mathrm{m}^{3}$ incremental increase in $\mathrm{PM}_{2.5}$ leading to a $5 \%, 7 \%$, or $15 \%$ increased risk of ASD in newborns depending on the model used ${ }^{24}$. Prenatal exposure to nitrogen oxides $\left(\mathrm{NO}_{\mathrm{X}}\right)$, mainly from traffic, has also been associated with an increased risk of ASD among children in a low-exposure setting 25 .

ASD development is poorly understood, but mounting evidence has emphasized the importance of genetic and environmental factors ${ }^{26}$. With the latter being modifiable, research on the role of the environment, particularly air pollution, has increased. Suggested biological mechanisms include oxidative stress, inflammation (neuro-inflammation and systemic inflammation), neurotoxicity, and endocrine disruption ${ }^{27-30}$. Animal models also support developmental neurotoxicity as a plausible pathway ${ }^{31,32}$, and one such study has demonstrated ASD traits in mice following prenatal exposure to diesel exhaust ${ }^{33}$. 
Regarding the underlying pathways of prenatal air pollution exposure in particular, a recent study of maternal serum samples indicated that high exposure to traffic-related air pollution during pregnancy disturbed metabolic pathways and mitochondrial function among mothers with children that developed $\mathrm{ASD}^{34}$. Moreover, short- and long-term species of $\mathrm{PM}_{2.5}$ that can be traced to wood burning, including ultra-fine particulate matter (UFP), black carbon (BC), potassium, and copper, have been shown to negatively affect several metabolic pathways involved in oxidative stress and inflammation ${ }^{35}$, but this was not specific to ASD development. Additionally, observational and controlled human exposure studies have found positive associations between Delta-C exposure, a marker for wood smoke, and inflammation ${ }^{36}$ and systemic oxidative stress ${ }^{37}$, respectively. Others have not found associations, however ${ }^{36,38,39}$.

Although air pollution has been demonstrated to significantly affect neurodevelopment and contribute to ASD according to the latest systematic reviews and meta-analyses ${ }^{23,24}$, no study could be identified considering these associations with respect to source-specific exposure. As the sources of locally produced air pollution may vary from setting to setting and each source can have unique effects on human health, previous research has encouraged more source-apportionment studies of $\mathrm{PM}^{40}$. Road traffic is often one of the largest contributors to PM emissions and is predicted to increase ${ }^{24}$, thus, exploring the various sources of traffic-related PM can provide a better understanding of their individual impacts on public health. Additionally, too few epidemiological studies investigating the association between ambient wood smoke from residential wood combustion and health exist to date ${ }^{41}$, and none consider ASD. Indeed, a review on the health impacts of wood burning emissions cited the need for more studies on non-respiratory pediatric outcomes, with better exposure assessment and proper adjustment for confounding ${ }^{42}$. The knowledge gleaned from source-specific findings can be used to inform air quality policy on all potentially detrimental emission sources.

The aim of the present study was to investigate source-specific ambient $\mathrm{PM}_{2.5}$ in association with autism spectrum disorders in a low-exposure setting where air pollution concentrations generally comply with the current European air quality guidelines ${ }^{43}$.

\section{Methods}

\subsection{Study setting and study population}

This study was undertaken in Scania (Skåne), the southernmost county in Sweden, which had a total population of approximately 1.4 million at the end of 2019. The study population is composed of the Maternal Air Pollution in Southern Sweden (MAPSS) cohort, containing data on 48,571 singleton births from 1999 to 2009 in Scania. A detailed description of this cohort has been provided previously by Malmqvist et al., $2017^{44}$. In short, MAPSS utilizes a high-quality local birth register with $98 \%$ coverage of all births in Scania and includes important data on the women's obstetric history, risk factors, and other 
relevant covariates. The birth register, Perinatal Revision Syd (PRS), has a wide catchment area of hospitals in Malmö, Lund, and Trelleborg (Figure 1).

Using each woman's unique personal identification number, PRS was linked to air pollution levels at each residential address as well as demographic information and socioeconomic characteristics obtained from Statistics Sweden. This data combined constitutes MAPSS. See Figure 2 for a detailed flowchart of the study population for the present paper.

\subsection{Exposure assessment}

A Gaussian plume air dispersion model was developed to extrapolate the monthly mean air pollution concentrations in Scania, Sweden, during the study period ${ }^{45}$. This flat, two-dimensional dispersion model was constructed using the software program ENVIMAN, a locally adjusted version of the American Meteorological Society/Environmental Protection Agency Regulatory Model (AERMOD) by the United States Environmental Protection Agency ${ }^{46}$. Annual mean source specific $\mathrm{PM}_{2.5}$ concentrations were modelled for two years (2000 and 2011) at $100 \mathrm{~m}$ by $100 \mathrm{~m}$ spatial resolution, while monthly mean concentrations of all local $\mathrm{PM}_{2.5}$ were available from a previous study ${ }^{45}$. The interpolation of the years and months between them was based on an atmospheric ventilation index using year- and monthspecific meteorological parameters ${ }^{45}$.

Emission sources included aviation, industries, major energy and heat producers, railroads, road traffic, shipping, small-scale residential heating, non-road vehicles, and local emissions in Zealand, Denmark. Aviation emission data were obtained from Scandinavian airports' annual environmental reports. Industry and energy production emissions were reported by relevant entities within the study area. Railroads in Sweden are mainly electric; therefore, railroad emissions were estimated using the fuel consumption of the few operational diesel engine freight trains both while in transit on railway lines and while static at railway stations ${ }^{44}$. Detailed data on fuel sources, types of vehicles, and speed limits was gathered from the Swedish Road Administration to account for emissions from road traffic. Furthermore, the emission factors developed by the Handbook of Emission Factors for Road Transport (HBEFA), version $3.2^{47}$ were applied to the vehicle types when estimating the emissions of $\mathrm{PM}_{2.5}$ from the local traffic emission sources (road segments). Shipping emissions for the year 2000 were estimated by Gustafsson ${ }^{46}$, and 2011 emissions were described by Project Shipair ${ }^{48}$. Regarding small-scale residential heating, the frequency of for example stove-use was estimated using chimney sweeping records from the National Rescue Agency ${ }^{46}$. Emissions from non-road vehicles were derived from a report by the Swedish Environmental Research Institute, IVL. Scania's proximity to the industrial island Zealand and the area's prevailing westerly winds warrants the inclusion of their local emissions in the dispersion model ${ }^{45}$.

Temporal and meteorological variations of air pollution, were, furthermore, addressed through the atmospheric ventilation index using a complex method developed by the SCAC project ${ }^{45}$. The modeled source specific annual means (for 2000 and 2011) were transformed to monthly means based on monthly profiles from the developed model (emission database). For all-source local $\mathrm{PM}_{2.5}$ monthly 
means were already available. By applying the atmospheric ventilation index to the start and end values, monthly means were interpolated for each $100 \mathrm{~m}$ by $100 \mathrm{~m}$ grid cell. Those monthly mean levels approximately corresponded to the calendar months of the women's pregnancies.

The geographical coordinates of each woman's residential address were obtained from Statistics Sweden, linked to MAPSS, and used to calculate individual exposure. Changes in residency were only updated at the end of the calendar year. Exposure estimates for every gestational month were, therefore, based on the nearest available time: January-June coordinates from the end of the previous year and July-December coordinates from the end of the current year. Moreover, if $67 \%$ or more of monthly exposure data was non-missing, the pregnancy exposure overall was designated as non-missing.

Because this study examined locally emitted $\mathrm{PM}_{2.5}$, regional background levels (i.e., those generated elsewhere that have traveled into the study area) were not considered. As regional background emissions typically comprise the majority of total PM, investigating only local PM concentrations results in seemingly low levels. However, the spatial contrast for regional background levels in this study area is low $^{45}$. Because of this, effect estimates would still describe contrasts in local exposures even if regional levels were included. The interpretation of the estimates becomes somewhat different though, illustrating the effect of local contrasts rather than the aggregate effect of total PM, which is more traditionally studied.

Four measures of locally emitted $\mathrm{PM}_{2.5}$ were investigated: all-source $\mathrm{PM}_{2.5}$, tailpipe exhaust, vehicle wearand-tear, and small-scale residential heating. Wear-and-tear comprises the air pollution generated from vehicles' brakes and tires, for example, and small-scale residential heating mainly consists of wood burning. All other source contributions (aviation, industries, major energy and heat producers, railroads, shipping, non-road vehicles, etc.) were very small. Therefore, these were not examined separately but were incorporated into the all-source $\mathrm{PM}_{2.5}$ category.

\subsection{Outcome assessment}

The outcome of interest in this study was autism spectrum disorders (ASD). When a child is suspected of having autism in Scania, they are referred to the Departments of Child and Adolescent Psychiatry and are examined by a multidisciplinary team ${ }^{25}$. These evaluations utilize both the Autism Diagnostic Observation Schedule-Generic (ADOS-G) ${ }^{49}$ and the Autism Diagnostic Interview-Revised (ADI-R) ${ }^{50}$ for most (75\%) cases. Finally, an ASD diagnosis is assigned according to the International Classification of Mental and Behavioral Disorders version 10 (ICD-10) and entered into the Skåne Healthcare Database (SHR). The outcome data used in this study was extracted from SHR.

ASD was identified by ICD-10 diagnosis codes starting with F84, which comprise all pervasive developmental disorders. This is characterized by one or more of the following areas of neurodivergence: qualitative variations in patterns of communication; difficulties with reciprocal social interactions; and a restricted, repetitive collection of behaviors and interests. Children usually receive a diagnosis around 7 years of age ${ }^{51}$; however, symptoms do not have to be present by a specified age. 
A further restriction of autism cases to childhood autism (ICD-10 code F84.0), where symptoms within all three areas of neurodivergence must be present before the age of 3 years, was also applied.

\subsection{Covariates}

The risk factors for autism development, identified a priori, that were considered for this study were maternal age $(\leq 19,20-34, \geq 35)$, parity $(1,2,3$, or $\geq 4)$, pre-pregnancy body mass index $(\mathrm{BMl} ;<18.5$, $18.5-24.9,25-29.9, \geq 30 \mathrm{~kg} / \mathrm{m}^{2}$ ), smoking status at first antenatal visit (non-smoker, 1-9 cigarettes/day, $\geq 10$ cigarettes/day), as well as sex of the child (male/female) and birth year (1999-2009).

Socioeconomic status (SES) predictors were also incorporated to control for potential confounding; these included maternal birth country (Sweden, Europe, other), maternal education level (pre-secondary, secondary, and post-secondary), annual household disposable income (quartiles), and neighborhoodlevel SES. Neighborhood-level SES was used a continuous measure, and captures the proportion of inhabitants in the neighborhood with "low economic standard", which is defined by Statistics Sweden as the number of people living in a household with an economic standard that is less than $60 \%$ of the national median value ${ }^{52}$. Other covariates considered in sensitivity analyses include birth month, birth weight, low birth weight.

\subsection{Statistical methods}

The main analysis applied binary logistic regression models with ASD as the outcome in a univariate analysis and two multivariate analyses. The first was a partially adjusted model which included only variables that were statistically significant in the models where total (local) $\mathrm{PM}_{2.5}$ was investigated, namely, parity, pre-pregnancy body mass index, sex of the child, and birth year. In addition to these variables, the second multivariate model, hereafter referred to as the fully adjusted model, also included maternal age, smoking status at first antenatal visit, maternal birth country, maternal education level, annual household disposable income, and neighborhood-level SES. As correlation coefficients between the different sources of $\mathrm{PM}_{2.5}$ (Supplementary Table S1, Supplementary Information) were very high, a single pollutant model was used. Linear exposure trends were assessed for atmospheric particles, using a continuous increment of a $1 \mu \mathrm{g} / \mathrm{m}^{3}$ increase in local $\mathrm{PM}_{2.5}$ levels.

Several secondary analyses were conducted. A subgroup analysis restricted analyses to childhood autism (ICD-10 diagnosis code F84.0) only. A subpopulation analysis including only Swedish-born women was also performed, with ASD as the outcome. Finally, exposure during the first year of life, instead of the pregnancy period, was explored for ASD. All utilized all-source $\mathrm{PM}_{2.5}$ as the exposure.

To address uncertainties, three sensitivity analyses were conducted. One adjusted for birth month and excluded children with a low birth weight and children born to mothers born outside Sweden. Finally, the main analysis was rerun with imputed exposure data, using the Expectation-Maximization algorithm. These sensitivity analyses were conducted for all-source $\mathrm{PM}_{2.5}$ with ASD as the outcome. 
All statistical analyses were carried out using SPSS version 27. Odds ratios (OR) and their corresponding $95 \%$ confidence intervals $(\mathrm{Cl})$ were reported for all analyses.

\subsection{Ethical approval}

The Lund University Ethical Committee approved this study prior to its realization (permission number 2014/696 and amendment 2016/211).

\section{Results}

The relationship between the exposures, covariates and ASD are documented in Table 1. There were clear associations between ASD and various markers of socioeconomic status, smoking intensity, BMI and $\mathrm{PM}_{2.5}$ exposure.

Table 1. Mean and standard deviation (SD) of local $\mathrm{PM}_{2.5}$ concentrations $\left(\mu \mathrm{g} / \mathrm{m}^{3}\right)$ during pregnancy for autism spectrum disorder (ASD) and covariates ( $N=46,340$ in total). 


\begin{tabular}{|c|c|c|c|c|c|c|}
\hline & $\begin{array}{l}\text { Number of } \\
\text { subjects* }\end{array}$ & $\begin{array}{l}\text { All- } \\
\text { source } \\
\mathrm{PM}_{2.5}\end{array}$ & $\begin{array}{l}\text { Small- } \\
\text { scale } \\
\text { heating }\end{array}$ & $\begin{array}{l}\text { Tailpipe } \\
\text { exhaust }\end{array}$ & $\begin{array}{l}\text { Vehicle } \\
\text { wear-and- } \\
\text { tear }\end{array}$ & $\begin{array}{l}\mathrm{ASD}^{+} \\
(\%)\end{array}$ \\
\hline$N$ & 46,340 & 36,713 & 30,781 & 30,694 & 33,247 & $\begin{array}{l}425 \\
(0.9)\end{array}$ \\
\hline Total & $36,713^{\ddagger}$ & $\begin{array}{l}1.50 \\
(0.71)\end{array}$ & $\begin{array}{l}0.58 \\
(0.37)\end{array}$ & $\begin{array}{l}0.15 \\
(0.09)\end{array}$ & $0.33(0.24)$ & \\
\hline \multicolumn{7}{|l|}{ ASD } \\
\hline No & 46,005 & $\begin{array}{l}1.50 \\
(0.71)\end{array}$ & $\begin{array}{l}0.58 \\
(0.37)\end{array}$ & $\begin{array}{l}0.15 \\
(0.09)\end{array}$ & $0.33(0.24)$ & - \\
\hline Yes & 335 & $\begin{array}{l}1.65 \\
(0.68)\end{array}$ & $\begin{array}{l}0.65 \\
(0.39)\end{array}$ & $\begin{array}{l}0.16 \\
(0.09)\end{array}$ & $0.37(0.24)$ & - \\
\hline \multicolumn{7}{|c|}{ Sex of the child } \\
\hline Male & 23,878 & $\begin{array}{l}1.51 \\
(0.71)\end{array}$ & $\begin{array}{l}0.58 \\
(0.37)\end{array}$ & $\begin{array}{l}0.15 \\
(0.09)\end{array}$ & $0.33(0.24)$ & $\begin{array}{l}325 \\
(1.4)\end{array}$ \\
\hline Female & 22,552 & $\begin{array}{l}1.50 \\
(0.71)\end{array}$ & $\begin{array}{l}0.58 \\
(0.37)\end{array}$ & $\begin{array}{l}0.15 \\
(0.09)\end{array}$ & $0.33(0.23)$ & $\begin{array}{l}100 \\
(0.4)\end{array}$ \\
\hline \multicolumn{7}{|c|}{$\begin{array}{l}\text { Household } \\
\text { disposable income level } \\
\text { (quartiles) }\end{array}$} \\
\hline Lowest & 11,606 & $\begin{array}{l}1.77 \\
(0.68)\end{array}$ & $\begin{array}{l}0.66 \\
(0.39)\end{array}$ & $\begin{array}{l}0.17 \\
(0.09)\end{array}$ & $0.40(0.24)$ & $\begin{array}{l}131 \\
(1.1)\end{array}$ \\
\hline Lower middle & 11,716 & $\begin{array}{l}1.64 \\
(0.72)\end{array}$ & $\begin{array}{l}0.65 \\
(0.38)\end{array}$ & $\begin{array}{l}0.16 \\
(0.09)\end{array}$ & $0.36(0.24)$ & $\begin{array}{l}124 \\
(1.1)\end{array}$ \\
\hline Higher middle & 11,768 & $\begin{array}{l}1.37 \\
(0.68)\end{array}$ & $\begin{array}{l}0.54 \\
(0.33)\end{array}$ & $\begin{array}{l}0.13 \\
(0.09)\end{array}$ & $0.29(0.23)$ & $\begin{array}{l}97 \\
(0.8)\end{array}$ \\
\hline Highest & 11,326 & $\begin{array}{l}1.25 \\
(0.62)\end{array}$ & $\begin{array}{l}0.47 \\
(0.32)\end{array}$ & $\begin{array}{l}0.12 \\
(0.08)\end{array}$ & $0.28(0.22)$ & $\begin{array}{l}73 \\
(0.6)\end{array}$ \\
\hline \multicolumn{7}{|c|}{$\begin{array}{l}\text { Maternal education } \\
\text { (years) }\end{array}$} \\
\hline$\leq 9$ & 5,920 & $\begin{array}{l}1.71 \\
(0.70)\end{array}$ & $\begin{array}{l}0.65 \\
(0.39)\end{array}$ & $\begin{array}{l}0.17 \\
(0.09)\end{array}$ & $0.38(0.24)$ & $\begin{array}{l}55 \\
(0.9)\end{array}$ \\
\hline $10-12$ & 19,404 & $\begin{array}{l}1.45 \\
(0.72)\end{array}$ & $\begin{array}{l}0.56 \\
(0.36)\end{array}$ & $\begin{array}{l}0.14 \\
(0.09)\end{array}$ & $0.31(0.23)$ & $\begin{array}{l}185 \\
(1.9)\end{array}$ \\
\hline $13-16$ & 18,481 & $\begin{array}{l}1.47 \\
(0.68)\end{array}$ & $\begin{array}{l}0.56 \\
(0.35)\end{array}$ & $\begin{array}{l}0.14 \\
(0.09)\end{array}$ & $0.33(0.23)$ & $\begin{array}{l}158 \\
(0.9)\end{array}$ \\
\hline$>16$ & 736 & $\begin{array}{l}1.38 \\
(0.61)\end{array}$ & $\begin{array}{l}0.57 \\
(0.36)\end{array}$ & $\begin{array}{l}0.12 \\
(0.09)\end{array}$ & $0.20(0.33)$ & $\begin{array}{l}10 \\
(1.4)\end{array}$ \\
\hline
\end{tabular}




\begin{tabular}{|c|c|c|c|c|c|c|}
\hline$\leq 30$ & 25,234 & $\begin{array}{l}1.55 \\
(0.71)\end{array}$ & $\begin{array}{l}0.59 \\
(0.37)\end{array}$ & $\begin{array}{l}0.15 \\
(0.09)\end{array}$ & $0.35(0.24)$ & $\begin{array}{l}243 \\
(1)\end{array}$ \\
\hline $31-34$ & 12,420 & $\begin{array}{l}1.45 \\
(0.69)\end{array}$ & $\begin{array}{l}0.56 \\
(0.36)\end{array}$ & $\begin{array}{l}0.14 \\
(0.09)\end{array}$ & $0.32(0.23)$ & $\begin{array}{l}94 \\
(0.8)\end{array}$ \\
\hline$\geq 35$ & 8,876 & $\begin{array}{l}1.44 \\
(0.69)\end{array}$ & $\begin{array}{l}0.56 \\
(0.37)\end{array}$ & $\begin{array}{l}0.14 \\
(0.09)\end{array}$ & $0.31(0.22)$ & $\begin{array}{l}88 \\
(1.0)\end{array}$ \\
\hline \multicolumn{7}{|c|}{ Maternal smoking } \\
\hline Non-smoker & 38,775 & $\begin{array}{l}1.51 \\
(0.71)\end{array}$ & $\begin{array}{l}0.59 \\
(0.37)\end{array}$ & $\begin{array}{l}0.15 \\
(0.09)\end{array}$ & $0.34(0.24)$ & $\begin{array}{l}349 \\
(0.9)\end{array}$ \\
\hline $1-9 \mathrm{cig} / \mathrm{day}$ & 3,136 & $\begin{array}{l}1.53 \\
(0.71)\end{array}$ & $\begin{array}{l}0.60 \\
(0.37)\end{array}$ & $\begin{array}{l}0.15 \\
(0.09)\end{array}$ & $0.33(0.23)$ & $\begin{array}{l}33 \\
(1.1)\end{array}$ \\
\hline$\geq 10 \mathrm{cig} /$ day & 1,320 & $\begin{array}{l}1.60 \\
(0.74)\end{array}$ & $\begin{array}{l}0.64 \\
(0.39)\end{array}$ & $\begin{array}{l}0.15 \\
(0.09)\end{array}$ & $0.34(0.23)$ & $\begin{array}{l}17 \\
(1.3)\end{array}$ \\
\hline \multicolumn{7}{|c|}{ Maternal BMI $\left(\mathrm{kg} / \mathrm{m}^{2}\right)$} \\
\hline$<18.5$ & 1,095 & $\begin{array}{l}1.58 \\
(0.70)\end{array}$ & $\begin{array}{l}0.62 \\
(0.38)\end{array}$ & $\begin{array}{l}0.16 \\
(0.09)\end{array}$ & $0.36(0.25)$ & $\begin{array}{l}15 \\
(1.4)\end{array}$ \\
\hline $18.5 \leq 25$ & 25,570 & $\begin{array}{l}1.53 \\
(0.70)\end{array}$ & $\begin{array}{l}0.59 \\
(0.37)\end{array}$ & $\begin{array}{l}0.15 \\
(0.09)\end{array}$ & $0.34(0.24)$ & $\begin{array}{l}198 \\
(0.8)\end{array}$ \\
\hline $25 \leq 30$ & 9,953 & $\begin{array}{l}1.50 \\
(0.72)\end{array}$ & $\begin{array}{l}0.58 \\
(0.37)\end{array}$ & $\begin{array}{l}0.15 \\
(0.09)\end{array}$ & $0.33(0.24)$ & $\begin{array}{l}106 \\
(1.1)\end{array}$ \\
\hline$\geq 30$ & 4,214 & $\begin{array}{l}1.51 \\
(0.74)\end{array}$ & $\begin{array}{l}0.57 \\
(0.37)\end{array}$ & $\begin{array}{l}0.15 \\
(0.09)\end{array}$ & $0.33(0.24)$ & $\begin{array}{l}57 \\
(1.4)\end{array}$ \\
\hline \multicolumn{7}{|c|}{ Maternal birth country } \\
\hline Sweden & 32,056 & $\begin{array}{l}1.38 \\
(0.70)\end{array}$ & $\begin{array}{l}0.54 \\
(0.35)\end{array}$ & $\begin{array}{l}0.13 \\
(0.09)\end{array}$ & $0.29(0.23)$ & $\begin{array}{l}296 \\
(0.9)\end{array}$ \\
\hline Europe & 6,275 & $\begin{array}{l}1.71 \\
(0.68)\end{array}$ & $\begin{array}{l}0.63 \\
(0.38)\end{array}$ & $\begin{array}{l}0.17 \\
(0.09)\end{array}$ & $0.40(0.24)$ & $\begin{array}{l}61 \\
(1.0)\end{array}$ \\
\hline Other & 8,097 & $\begin{array}{l}1.86 \\
(0.59)\end{array}$ & $\begin{array}{l}0.69 \\
(0.39)\end{array}$ & $\begin{array}{l}0.19 \\
(0.08)\end{array}$ & $0.45(0.22)$ & $\begin{array}{l}68 \\
(0.8)\end{array}$ \\
\hline \multicolumn{7}{|l|}{ Parity } \\
\hline $1^{\text {st }}$ child & 22,407 & $\begin{array}{l}1.56 \\
(0.72)\end{array}$ & $\begin{array}{l}0.59 \\
(0.37)\end{array}$ & $\begin{array}{l}0.15 \\
(0.09)\end{array}$ & $0.36(0.25)$ & $\begin{array}{l}238 \\
(1.1)\end{array}$ \\
\hline $2^{\text {nd }}$ child & 15,207 & $\begin{array}{l}1.41 \\
(0.68)\end{array}$ & $\begin{array}{l}0.56 \\
(0.35)\end{array}$ & $\begin{array}{l}0.14 \\
(0.08)\end{array}$ & $0.30(0.22)$ & $\begin{array}{l}110 \\
(0.7)\end{array}$ \\
\hline $3^{\text {rd }}$ child & 5,718 & $\begin{array}{l}1.43 \\
(0.69)\end{array}$ & $\begin{array}{l}0.56 \\
(0.36)\end{array}$ & $\begin{array}{l}0.14 \\
(0.08)\end{array}$ & $0.29(0.21)$ & $\begin{array}{l}55 \\
(1.0)\end{array}$ \\
\hline$\geq 4^{\text {th }}$ child & 3,098 & $\begin{array}{l}1.66 \\
(0.72)\end{array}$ & $\begin{array}{l}0.65 \\
(0.41)\end{array}$ & $\begin{array}{l}0.16 \\
(0.09)\end{array}$ & $0.37(0.24)$ & $\begin{array}{l}22 \\
(0.7)\end{array}$ \\
\hline
\end{tabular}




\begin{tabular}{|c|c|c|c|c|c|c|}
\hline \multicolumn{7}{|c|}{ Birth year } \\
\hline 2000 & 3,792 & $\begin{array}{l}1.43 \\
(0.65)\end{array}$ & $\begin{array}{l}0.66 \\
(0.32)\end{array}$ & $\begin{array}{l}0.15 \\
(0.08)\end{array}$ & $0.24(0.15)$ & $\begin{array}{l}32 \\
(0.8)\end{array}$ \\
\hline 2001 & 3,960 & $\begin{array}{l}1.65 \\
(0.76)\end{array}$ & $\begin{array}{l}0.80 \\
(0.43)\end{array}$ & $\begin{array}{l}0.15 \\
(0.09)\end{array}$ & $0.29(0.18)$ & $\begin{array}{l}43 \\
(1.1)\end{array}$ \\
\hline 2002 & 4,445 & $\begin{array}{l}1.50 \\
(0.66)\end{array}$ & $\begin{array}{l}0.64 \\
(0.29)\end{array}$ & $\begin{array}{l}0.13 \\
(0.07)\end{array}$ & $0.27(0.17)$ & $\begin{array}{l}51 \\
(1.1)\end{array}$ \\
\hline 2003 & 4,455 & $\begin{array}{l}1.70 \\
(0.80)\end{array}$ & $\begin{array}{l}0.77 \\
(0.42)\end{array}$ & $\begin{array}{l}0.17 \\
(0.10)\end{array}$ & $0.33(0.22)$ & $\begin{array}{l}45 \\
(1.0)\end{array}$ \\
\hline 2004 & 4,561 & $\begin{array}{l}1.53 \\
(0.69)\end{array}$ & $\begin{array}{l}0.64 \\
(0.31)\end{array}$ & $\begin{array}{l}0.15 \\
(0.09)\end{array}$ & $0.32(0.22)$ & $\begin{array}{l}46 \\
(1.0)\end{array}$ \\
\hline 2005 & 4,747 & $\begin{array}{l}1.55 \\
(0.73)\end{array}$ & $\begin{array}{l}0.34 \\
(0.20)\end{array}$ & $\begin{array}{l}0.11 \\
(0.07)\end{array}$ & $0.27(0.19)$ & $\begin{array}{l}33 \\
(0.7)\end{array}$ \\
\hline 2006 & 4,135 & $\begin{array}{l}1.58 \\
(0.71)\end{array}$ & $\begin{array}{l}0.44 \\
(0.36)\end{array}$ & $\begin{array}{l}0.16 \\
(0.10)\end{array}$ & $0.42(0.29)$ & $\begin{array}{l}40 \\
(1.0)\end{array}$ \\
\hline 2007 & 4,625 & $\begin{array}{l}1.26 \\
(0.57)\end{array}$ & $\begin{array}{l}0.31 \\
(0.21)\end{array}$ & $\begin{array}{l}0.15 \\
(0.09)\end{array}$ & $0.38(0.27)$ & $\begin{array}{l}35 \\
(0.8)\end{array}$ \\
\hline 2008 & 4,664 & $\begin{array}{l}1.25 \\
(0.56)\end{array}$ & $\begin{array}{l}0.27 \\
(0.13)\end{array}$ & $\begin{array}{l}0.12 \\
(0.07)\end{array}$ & $0.37(0.27)$ & $\begin{array}{l}32 \\
(0.7)\end{array}$ \\
\hline 2009 & 3,872 & $\begin{array}{l}1.44 \\
(0.67)\end{array}$ & $\begin{array}{l}0.38 \\
(0.21)\end{array}$ & $\begin{array}{l}0.15 \\
(0.10)\end{array}$ & $0.43(0.30)$ & $\begin{array}{l}32 \\
(0.8)\end{array}$ \\
\hline
\end{tabular}

$\mathrm{PM}_{2.5}$ : Particulate matter with a diameter of <2.5 $\mu \mathrm{m}$. Cig: Cigarette. BMI: Body mass index. ${ }^{*}$ Total population. ${ }^{\dagger}$ International Classification of Mental and Behavioral Disorders version 10 (ICD-10) diagnoses codes beginning with F84 (given for total population). ${ }^{\ddagger}$ Given for all-source $\mathrm{PM}_{2.5}$ ( $\mathrm{N}$ may vary slightly by $\mathrm{PM}_{2.5}$ source). **Measured as annual disposable income.

All-source $\mathrm{PM}_{2.5}$ exposure during pregnancy was associated with ASD, with ORs of 1.28 (95\% Cl: 1.09$1.49)$ in the partially adjusted model and 1.23 (95\% Cl: $1.02-1.48)$ in the fully adjusted model (Table 2 ). Concerning the road traffic-related sources, both tailpipe exhaust and vehicle wear-and-tear were associated with ASD: ORs in the partially adjusted model were 6.71 (95\% Cl: 1.86-34.30) and 1.96 (95\% $\mathrm{Cl}: 1.21-3.17)$, respectively. An association was also found for exposure to $\mathrm{PM}_{2.5}$ from small-scale residential heating and ASD in the partially adjusted model, with an OR of 1.53 (95\% Cl: 1.09-2.14). In the fully adjusted models, however, none of the three source specific exposure ORs were statistically significant, although the point estimates remained high.

Table 2. Odds ratios (OR) and their $95 \%$ confidence intervals $(\mathrm{Cl})$ of autism spectrum disorders (ASD) associated with $1 \mu \mathrm{g} / \mathrm{m}^{3}$ increase in concentration of various components of the local contribution to $\mathrm{PM}_{2.5}$ during pregnancy. 


\begin{tabular}{|lllllll|}
\hline & \multicolumn{2}{l}{ Crude model } & \multicolumn{2}{l}{${\text { Main } \text { model }^{*}}^{2}$} & \multicolumn{2}{l|}{ Fully adjusted $^{\dagger}$} \\
& OR & $(95 \% \mathrm{Cl})$ & OR & $(95 \% \mathrm{Cl})$ & OR & $(95 \% \mathrm{Cl})$ \\
\hline All-source $\mathrm{PM}_{2.5}$ & 1.32 & $(1.14-1.52)$ & 1.28 & $(1.09-1.49)$ & 1.23 & $(1.02-1.48)$ \\
\hline Tailpipe exhaust & 8.32 & $(2.51-27.6)$ & 6.71 & $(1.86-24.3)$ & 4.47 & $(0.96-20.9)$ \\
\hline Vehicle wear-and-tear & 1.84 & $(1.20-2.84)$ & 1.96 & $(1.21-3.17)$ & 1.66 & $(0.94-2.93)$ \\
\hline Small-scale heating & 1.69 & $(1.27-2.26)$ & 1.53 & $(1.09-2.14)$ & 1.27 & $(0.86-1.88)$ \\
\hline
\end{tabular}

*Adjusted for maternal parity, pre-pregnancy BMI, sex of the child, and birth year. ${ }^{\dagger}$ Adjusted for maternal parity, pre-pregnancy BMI, sex of the child, birth year, maternal age, smoking status, birth country, education, income, and neighborhood-level SES. Number $(\mathrm{N})$ of women included in each model: Crude=36,713; Main=32,045; Fully adjusted=26,773 and given for all-source $\mathrm{PM}_{2.5}(\mathrm{~N}$ may vary slightly by $\mathrm{PM}_{2.5}$ source).

Results for the subgroup analysis on childhood autism, reported here for all-source $\mathrm{PM}_{2.5}$ in fully adjusted models, were similar but somewhat less precise compared to ASD, with an OR of 1.28 (95\% Cl: 1.031.59). The subpopulation analysis of Swedish-born women's exposure during pregnancy typically resulted in lower ORs compared to the entire study population: 1.13 (95\% $\mathrm{Cl}: 0.91-1.40)$. Associations between all four $\mathrm{PM}_{2.5}$ sources during the first year of life and ASD were generally less pronounced than those seen for exposure during pregnancy; for example, the OR for all-source $\mathrm{PM}_{2.5}$ was $1.17(95 \% \mathrm{Cl}$ : $0.96-1.42)$.

Additionally, when excluding LBW babies, the OR was 1.27 (95\% Cl: 1.05-1.53). The sensitivity analysis using imputed exposure data resulted in higher precision in the estimates, but the overall effect estimates were only marginally altered (data not shown).

\section{Discussion}

In this population-based study from southern Sweden, associations were observed between exposure to all investigated local sources of $\mathrm{PM}_{2.5}$ (all-source $\mathrm{PM}_{2.5}$, tailpipe exhaust, vehicle wear-and-tear, and small-scale residential heating) during pregnancy and ASD.

Although only statistically significant in the partially adjusted models and not in the fully adjusted models, the results suggest that both local $\mathrm{PM}_{2.5}$ from tailpipe exhaust and vehicle wear-and-tear contribute to the observed associations with ASD. These results are in line with our previous study on prenatal exposure to ambient $\mathrm{NO}_{\mathrm{X}}$ concentrations and autism diagnosis using the same cohort (MAPSS), where children in the highest exposure quartile during the entire pregnancy period had a $40 \%$ greater risk of developing ASD compared to those in the lowest ${ }^{25}$. Under an assumption of a causal association between $\mathrm{PM}_{2.5}$ and childhood autism, a health impact assessment conducted for Scania identified $3 \%$ of 
autism cases to be attributable to locally emitted $\mathrm{PM}_{2.5}$, of which $\sim 30 \%$ is derived from traffic ${ }^{53}$. Outside our study setting, two case-control studies from California found $15 \%$ increased odds ${ }^{54}$ and just over double the risk ${ }^{55}$ of autism development due to traffic-related $\mathrm{PM}_{2.5}$ exposure during pregnancy.

However, previous studies conducted in Stockholm, Sweden, did not find associations between exposure to traffic-related air pollution during pregnancy and $A S D^{56,57}$. Reasons for this conflict could include that these studies considered $\mathrm{PM}_{10}$ and $\mathrm{NO}_{\mathrm{X}}$, while ours investigated $\mathrm{PM}_{2.5}$, which has been the pollutant with the most evidence for autism development in systematic reviews ${ }^{23,24}$. One register-based study also used "symptoms of neurodevelopmental disorders" as its outcome as opposed to physician-diagnosed ASD ${ }^{56}$. Similarly, a study using four European cohorts, including a Swedish one, explored autistic traits, both symptom within the borderline/clinical range and within the clinical range using validated cut-offs, but did not find an association with air pollution, even for $\mathrm{PM}_{2.5}$, using land-use regression models, with main predictor variables being traffic, space heating, and population/household density ${ }^{58}$. Interestingly, in neighboring Denmark, researchers found that exposure to traffic-related $\mathrm{NO}_{2}, \mathrm{PM}_{10}$, and $\mathrm{PM}_{2.5}$ in early infancy, not during pregnancy, was associated with autism ${ }^{59}$. While our study's emphasis is on the pregnancy period, exposure during the first year of life was also considered, and statistically significant associations with $\mathrm{ASD}$ and $\mathrm{PM}_{2.5}$ were found. However, associations for exposure during fetal life appeared stronger in our data (due to high correlation we could not investigate both fetal life and first year of life in the same statistical models). Systematic reviews have noted both the pregnancy period and postnatal periods as decisive exposure windows ${ }^{24}$.

To the best of our knowledge, no previous study has further separated $\mathrm{PM}_{2.5}$ from road traffic into tailpipe exhaust versus vehicle wear-and-tear and explored their associations with ASD.

No previous epidemiological studies investigating the effects of source-specific ambient wood smoke exposure from small-scale residential heating on autism development could be identified. Concerning neurodegenerative conditions in general, a longitudinal study in northern Sweden has indicated that $\mathrm{PM}_{2.5}$ from residential wood burning is associated with dementia incidence ${ }^{10}$. In an experimental study using a placental first trimester trophoblast cell line, exposure to wood smoke particles caused cytotoxicity and disrupted proliferation in exposed placenta cells, and particles detected inside cells caused structural damage to mitochondria and endoplasmic reticulum ${ }^{60}$. In line with this, sourceapportionment studies on pregnancy complications found increased concentrations of Delta-C (a marker for wood smoke) and BC during wintertime to be associated with greater odds of developing early-onset preeclampsia ${ }^{61}$. Similar studies exploring birth outcomes, however, reported that $\mathrm{PM}_{2.5}$ from ambient biomass burning (i.e., wood smoke) was associated with a lower risk of preterm birth ${ }^{16}$, low birth weight ${ }^{17}$, and stillbirth ${ }^{18}$. Authors attributed these findings to the high winter seasonality of biomass burning ${ }^{16}$; it being inversely associated with most of the other $\mathrm{PM}_{2.5}$ sources explored ${ }^{17}$; and its negative correlation with re-suspended soil in particular ${ }^{18}$. In our study, however, no inverse correlations were seen between small-scale residential heating and the other $\mathrm{PM}_{2.5}$ sources. 
Due to its unique chemical composition, $\mathrm{PM}_{2.5}$ derived from wood smoke may have varying toxicity compared to other sources of ambient $\mathrm{PM}_{2.5}$. Indeed, a 2003 review stated that studies including residential wood combustion as a major source of PM reported higher relative risks for adverse health outcomes compared to general ambient PM estimations ${ }^{62}$. Conversely, a 2018 review found that most source-apportionment studies reported PM from ambient biomass to be less detrimental to health than other sources; however, the wood smoke assessed was not necessarily derived from residential wood burning only ${ }^{40}$. A noted exception from Copenhagen observed stronger point estimates for $\mathrm{PM}_{10}$ apportioned to biomass (mainly wood burning) than $\mathrm{PM}_{10}$ derived from traffic ${ }^{63}$. Examining the impact of short-term exposure to $\mathrm{PM}_{2.5}$ from traffic and wood smoke on mortality, a study also found higher statistically significant risk-increases when 24-hour average concentrations were used and stronger increased risks for deaths occurring in the cold season, both of which better represent exposure from wood burning ${ }^{64}$. In the present study, emphasis is placed on the positive associations observed for all $\mathrm{PM}_{2.5}$ sources as opposed to comparing individual point estimates.

Despite the varying results in current literature, the continued inclusion of residential wood burning in source-specific air pollution epidemiology is pertinent, as it has been shown to be a significant source of ambient PM, particularly in the wintertime. According to a recent review, residential wood combustion in developed countries may even dominate $\mathrm{PM}_{2.5}$ and $\mathrm{PM}_{10}$ concentrations during colder seasons, contribute to more than $40 \%$ of organic carbon attached to $\mathrm{PM}_{2.5}$ and $\mathrm{PM}_{10}$, and influence ambient elemental carbon (EC) concentrations, which is correlated with $\mathrm{BC}^{65}$. In Sweden, specifically, recreational wood burning accounts for approximately $34 \%$ of small houses' heat demand ${ }^{66}$. In Scania, emissions from small-scale residential heating (mainly wood burning) have been calculated to comprise $12 \%$ of the total $\mathrm{PM}_{10}$, and $21 \%$ of the total soot in 2011 ; this source was even estimated to account for as much as $89 \%$ of the local $\mathrm{PM}_{2.5}$ concentrations in certain locations ${ }^{67}$. Moreover, wood burning's relative contribution to total EC was more than three times higher at a rural site outside of Gothenburg, Sweden, than an urban one ${ }^{68}$. Interestingly, authors found that the spatial variability of wood burning aerosols between the two sites was moderate, and that high fossil fuel emissions at the urban site decreased wood smoke's relative contribution ${ }^{68}$. This demonstrates that small-scale residential heating is also prominent in urban areas.

No studies could be identified that apportioned $\mathrm{PM}_{2.5}$ traffic emissions into its tailpipe exhaust versus vehicle wear-and-tear components and included autism spectrum disorder as an outcome, thus future ASD research should consider further source-specific separation. The subsequent findings would provide a more detailed understanding of how various $\mathrm{PM}_{2.5}$ sources affect human health. Such information could be used for government-level policy development regarding traffic-related air pollution, such as creating additional regulations on vehicle emissions, including European Emission Standards ${ }^{69}$, subsidizing electric vehicles ${ }^{70}$, establishing or revising low-emission zones within cities ${ }^{71,72}$, or improving protocols for tires, brakes, and road surfaces. Cities can also prioritize reducing traffic overall by investing in public transportation options and establishing safe, convenient active transportation options, such as 
a networks of bike lanes and well-maintained pedestrian sidewalks ${ }^{73,74}$. Outside of policy, such research can also inform priority areas for industry-level technological development.

Moreover, recent reviews ${ }^{40,42}$ on the health effects of wood combustion PM emissions are limited to mainly respiratory outcomes, with some cardiovascular and oncological outcomes included. As the proportion of $\mathrm{PM}_{2.5}$ attributable to residential wood burning can be substantial, future research should consider investigating additional health effects of this $\mathrm{PM}_{2.5}$ source ${ }^{42}$, especially the development of ASD where current literature is lacking. Additional source-apportionment studies on these associations are needed to substantiate our results. Recreational wood burning has been identified as a potential challenge toward air quality control due to the increase in woodstove use, particularly in urban areas according to a recent study evaluating the air pollution mitigation strategies of 10 European cities, including Malmö, Sweden ${ }^{75}$. As lighting recreational fires has been linked to positive emotional outcomes, such as decreasing stress and promoting joy ${ }^{76}$, pollution reduction from this source may need to rely on technological advancements, including combustion efficiency and heat exchanger technology ${ }^{65}$. The World Health Organization has also recommended "no burn" areas and "no wood burning" days policies as well as public information campaigns on energy efficiency ${ }^{77}$. Regardless of the mitigation method, the health impacts of reducing ambient $\mathrm{PM}_{2.5}$ and $\mathrm{PM}_{10}$ concentrations from residential wood burning in heaters and fireplaces, particularly in the wintertime, have been shown to be substantial ${ }^{78}$.

A key strength of this study is its large sample population derived from the MAPSS birth cohort, comprising $98 \%$ of all births occurring in the hospital catchment areas in Scania. With this, power and validity were increased. Utilizing a validated, high resolution dispersion model to estimate $\mathrm{PM}_{2.5}$ exposure was also an important strength that improved the reliability of results. Further, this dispersion model incorporates contributions from the various $\mathrm{PM}_{2.5}$ sources separately at baseline, which bypasses the difficult process of disentangling aggregated emissions. Obtaining the exact geographic coordinates of each woman's home residence was also vital for accurate exposure estimates at the individual level. An additional strength includes the thorough outcome assessment performed by Departments of Child and Adolescent Psychiatry. It should be mentioned, however, that the prerequisites for autism diagnosis have likely changed over time worldwide. A Swedish study, for instance, reported that the presence of significantly fewer autism symptoms appeared to be necessary to receive a clinical autism diagnosis for children diagnosed after preschool (ages 7-12) ${ }^{79}$. Nevertheless, any such trends in our data should not have affected the results because the analyses were adjusted for birth year.

Furthermore, health care systems in Sweden are tax-subsidized and used by virtually all residents, which increases the ability to identify physician-diagnosed cases of ASD and childhood autism (ICD-10 codes F84 and F84.0, respectively) and record them in high quality healthcare databases. With this, outcome misclassification, response-bias, recall-bias, and selection bias were avoided. Information on covariates incorporated into the adjusted models were similarly collected from well-managed, precise registers. These results are also considered generalizable to pregnant women in other similar study areas, where 
the sources of air pollution are comparable. The findings are relevant to both public health in general and the clinical setting specifically because they indicate that even small changes in locally produced $\mathrm{PM}_{2.5}$ concentrations can affect the risk of ASD among children. Finally, this study contributes evidence to an emerging research area investigating the health effects of local, source-specific air pollution exposure. Interestingly, accumulating evidence suggests that locally produced PM may be more hazardous to human health than regional, background concentrations ${ }^{80}$.

This study also has several limitations. For example, quite a large proportion of the study population had missing data on exposure, outcome, and/or covariates. Missing observations were able to be imputed in a sensitivity analysis, which resulted in increased precision of the corresponding estimates, as illustrated by narrower Cls, and relatively unchanged point estimates. Moreover, data on parental diagnoses was not available. Given that genetic factors account for a considerable part of the variation in autism development and emergence ${ }^{81}$, our results could partly be explained by heredity, if parents with autism were more likely to reside in areas characterized by higher levels of air pollution than parents without autism. Residual confounding due to other risk factors for autism, which are also associated with the exposure and not accounted for in our statistical models, may also be present. However, relevant risk factors have been included, which was based on current literature as well as a directed acyclic graph. Exposure misclassification may exist, as exposure was assessed at each woman's home residence, and participants' total exposure, including indoor, behaviour-related, transport-related and occupational, was not considered. This is deemed standard practice in air pollution epidemiology research, with the assumption that the resulting misclassification is non-differential. Results pertaining to the sourcespecific fractions of $\mathrm{PM}_{2.5}$ derived from tailpipe exhaust produced high point estimates and notably wide confidence intervals. The levels of locally produced $\mathrm{PM}_{2.5}$ in the study area are quite low, which makes the $1 \mu \mathrm{g} / \mathrm{m}^{3}$ increase in $\mathrm{PM}_{2.5}$ a sizeable increment and can contribute to high point estimates;

nevertheless, the study results emphasize that despite being low, the local contribution to $\mathrm{PM}_{2.5}$ may still have detrimental health effects. As the differences in ORs between the various sources were not formally tested and some sources are highly correlated with one another, direct comparisons between our sourcespecific risk estimates cannot be made. Instead, more studies, preferably in a multi-cohort setting, are needed to increase statistical power. When considering only mothers born in Sweden, the associations tended to be lower than for that of the entire study population. Findings from previous research on environmental injustice in Scania, showed that non-Swedish-born persons had higher odds of being exposed to greater concentrations of air pollution ${ }^{82}$. In Sweden, children born to women who emigrated from Sub-Saharan Africa and the Middle East are, furthermore, more commonly diagnosed with ASD ${ }^{51}$. Our results may also suggest that there is some residual confounding with respect to SES in the statistical models.

\section{Conclusion}

These findings add to current evidence that prenatal exposure to air pollution is associated with the risk of developing autism spectrum disorders and offers insight on these associations in a relatively low 
exposure setting. Additionally, this source-specific study indicates that each local $\mathrm{PM}_{2.5}$ source investigated (all-source, tailpipe exhaust, vehicle wear-and-tear, and small-scale residential heatingmainly wood burning) may be associated with the increased risk of ASD. This supports existing literature documenting the substantial health effects of locally produced particles, despite long-range, intransported contributions often comprising the majority of PM's total concentration.

\section{Declarations}

\section{Data Availability:}

The datasets generated during and/or analyzed during the current study are stored on a secure server and are not publicly available because they contain sensitive information (on health data, demographic characteristics, socioeconomic status) and, therefore, cannot be shared openly. However, they are available from Anna Oudin (anna.oudin@med.lu.se) on reasonable request.

\section{Acknowledgements:}

We would like to thank Susanna Gustafsson at the City of Malmö's Environmental Department for her work on exposure assessment and our colleague, Emilie Stroh, for creating the map in Figure 1. We are also grateful to the Swedish Research Council, Formas for providing funding (grant number 2017-00898) for this project.

\section{Author Contributions Statement:}

E.F. drafted and edited the manuscript, performed preliminary statistical analyses, and created Figure 2. E.M. helped conceive the study. R.R. was responsible for exposure assessment and data management. P.G. was responsible for the outcome data and validation together with this team. K.K. provided input on statistical analyses. A.O. conceived the study, performed final statistical analyses, and edited the manuscript. All authors reviewed and approved the final version of the manuscript.

\section{Additional Information:}

The authors declare no competing interests (financial or non-financial) as defined by Nature Research, or other interests that might be perceived to influence the results and/or discussion reported in this paper. The results/data/figures in this manuscript have not been published elsewhere, nor are they under consideration by another publisher.

\section{References}

1. Cohen, A. J. et al. Estimates and 25-year trends of the global burden of disease attributable to ambient air pollution: an analysis of data from the Global Burden of Diseases Study 2015. The Lancet 389, 1907-1918, doi:https://doi.org/10.1016/S0140-6736(17)30505-6 (2017). 
2. Vohra, K. et al. Global mortality from outdoor fine particle pollution generated by fossil fuel combustion: Results from GEOS-Chem. Environmental Research 195, 110754, doi:https://doi.org/10.1016/j.envres.2021.110754 (2021).

3. Matz, C. J. et al. Human health effects of traffic-related air pollution (TRAP): a scoping review protocol. Systematic Reviews 8, 223, doi:10.1186/s13643-019-1106-5 (2019).

4. Traffic-Related Air Pollution. 1 edn, (Elsevier Inc, 2020).

5. Kjällstrand, J. \& Petersson, G. Phenols and Aromatic Hydrocarbons in Chimney Emissions from Traditional and Modern Residential Wood Burning. Environmental Technology 22, 391-395, doi:10.1080/09593332208618271 (2001).

6. Zeglinski, M. R. et al. Soluble Wood Smoke Extract Promotes Barrier Dysfunction in Alveolar Epithelial Cells through a MAPK Signaling Pathway. Scientific Reports 9, 10027, doi:10.1038/s41598019-46400-8 (2019).

7. Huang, J. et al. Mercury (Hg) emissions from domestic biomass combustion for space heating. Chemosphere 84, 1694-1699, doi:https://doi.org/10.1016/j.chemosphere.2011.04.078 (2011).

8. Thurston, G., Awe, Y., Ostro, B. \& Sanchez-Triana, E. Are All Air Pollution Particles Equal? How Constituents and Sources of Fine Air Pollution Particles (PM2.5) Affect Health. (World Bank, Washington, DC, 2021).

9. Olsson, D., Johansson, C. \& Forsberg, B. Associations between Vehicle Exhaust Particles and Ozone at Home Address and Birth Weight. Int J Environ Res Public Health 17, 3836 (2020).

10. Oudin, A., Segersson, D., Adolfsson, R. \& Forsberg, B. Association between air pollution from residential wood burning and dementia incidence in a longitudinal study in Northern Sweden. PloS one 13, e0198283 (2018).

11. Olsson, D. et al. Early childhood exposure to ambient air pollution is associated with increased risk of paediatric asthma: An administrative cohort study from Stockholm, Sweden. Environment International 155, 106667 (2021).

12. Pedersen, M. et al. Ambient Air Pollution and Pregnancy-Induced Hypertensive Disorders. Hypertension 64, 494-500, doi:doi:10.1161/HYPERTENSIONAHA.114.03545 (2014).

13. Perera, F., Ashrafi, A., Kinney, P. \& Mills, D. Towards a fuller assessment of benefits to children's health of reducing air pollution and mitigating climate change due to fossil fuel combustion. Environmental Research 172, 55-72, doi:https://doi.org/10.1016/j.envres.2018.12.016 (2019).

14. Nääv, Å. et al. Urban PM2.5 Induces Cellular Toxicity, Hormone Dysregulation, Oxidative Damage, Inflammation, and Mitochondrial Interference in the HRT8 Trophoblast Cell Line. Frontiers in Endocrinology 11, doi:10.3389/fendo.2020.00075 (2020).

15. Dadvand, P. et al. Particulate air pollution and preeclampsia: a source-based analysis. Occup Environ Med 71, 570-577, doi:10.1136/oemed-2013-101693 (2014).

16. Ottone, M. et al. Source-related components of fine particulate matter and risk of adverse birth outcomes in Northern Italy. Environmental Research 186, 109564, doi:https://doi.org/10.1016/j.envres.2020.109564 (2020). 
17. $\mathrm{Ng}, \mathrm{C}$. et al. Source apportionment of fine particulate matter and risk of term low birth weight in California: Exploring modification by region and maternal characteristics. Science of The Total Environment 605-606, 647-654, doi:https://doi.org/10.1016/j.scitotenv.2017.06.053 (2017).

18. Ebisu, K., Malig, B., Hasheminassab, S., Sioutas, C. \& Basu, R. Cause-specific stillbirth and exposure to chemical constituents and sources of fine particulate matter. Environmental Research 160, 358364, doi:https://doi.org/10.1016/j.envres.2017.10.015 (2018).

19. Butler, A. S. \& Behrman, R. E. Preterm birth: causes, consequences, and prevention. (National Academies Press, 2007).

20. Barker, D., Eriksson, J., Forsén, T. \& Osmond, C. Fetal origins of adult disease: strength of effects and biological basis. International Journal of Epidemiology 31, 1235-1239, doi:10.1093/ije/31.6.1235 (2002).

21. Flores-Pajot, M.-C., Ofner, M., Do, M. T., Lavigne, E. \& Villeneuve, P. J. Childhood autism spectrum disorders and exposure to nitrogen dioxide, and particulate matter air pollution: A review and metaanalysis. Environmental Research 151, 763-776, doi:https://doi.org/10.1016/j.envres.2016.07.030 (2016).

22. Fu, P., Guo, X., Cheung, F. M. H. \& Yung, K. K. L. The association between PM2.5 exposure and neurological disorders: A systematic review and meta-analysis. Science of The Total Environment 655, 1240-1248, doi:https://doi.org/10.1016/j.scitotenv.2018.11.218 (2019).

23. Chun, H., Leung, C., Wen, S. W., McDonald, J. \& Shin, H. H. Maternal exposure to air pollution and risk of autism in children: A systematic review and meta-analysis. Environmental Pollution 256, 113307, doi:https://doi.org/10.1016/j.envpol.2019.113307 (2020).

24. Dutheil, F. et al. Autism spectrum disorder and air pollution: A systematic review and meta-analysis. Environmental Pollution 278, 116856, doi:https://doi.org/10.1016/j.envpol.2021.116856 (2021).

25. Oudin, A. et al. Prenatal exposure to air pollution as a potential risk factor for autism and ADHD. Environment international 133, 105149 (2019).

26. Volk, H. E. et al. Autism Spectrum Disorder: Interaction of Air Pollution with the MET Receptor Tyrosine Kinase Gene. Epidemiology 25, 44-47, doi:10.1097/ede.0000000000000030 (2014).

27. Block, M. L. \& Calderón-Garcidueñas, L. Air pollution: mechanisms of neuroinflammation and CNS disease. Trends in neurosciences 32, 506-516, doi:10.1016/j.tins.2009.05.009 (2009).

28. Costa, L. G., Chang, Y.-C. \& Cole, T. B. Developmental Neurotoxicity of Traffic-Related Air Pollution: Focus on Autism. Current Environmental Health Reports 4, 156-165, doi:10.1007/s40572-017-0135-2 (2017).

29. Costa, L. G. et al. Neurotoxicity of traffic-related air pollution. NeuroToxicology 59, 133-139, doi:https://doi.org/10.1016/j.neuro.2015.11.008 (2017).

30. Ha, S. Air pollution and neurological development in children. Dev Med Child Neurol 63, 374-381, doi:10.1111/dmcn.14758 (2021).

31. Allen, J. L. et al. Developmental neurotoxicity of inhaled ambient ultrafine particle air pollution: Parallels with neuropathological and behavioral features of autism and other neurodevelopmental 
disorders. Neurotoxicology 59, 140-154, doi:10.1016/j.neuro.2015.12.014 (2017).

32. Cory-Slechta, D. A. et al. The Impact of Inhaled Ambient Ultrafine Particulate Matter on Developing Brain: Potential Importance of Elemental Contaminants. Toxicologic Pathology 47, 976-992, doi:10.1177/0192623319878400 (2019).

33. Chang, Y.-C., Cole, T. B. \& Costa, L. G. Prenatal and early-life diesel exhaust exposure causes autismlike behavioral changes in mice. Particle and fibre toxicology 15, 18-18, doi:10.1186/s12989-0180254-4 (2018).

34. Kim, J. H. et al. Metabolomics analysis of maternal serum exposed to high air pollution during pregnancy and risk of autism spectrum disorder in offspring. Environmental Research 196, 110823, doi:https://doi.org/10.1016/j.envres.2021.110823 (2021).

35. Nassan, F. L. et al. Ambient PM2.5 species and ultrafine particle exposure and their differential metabolomic signatures. Environment International 151, 106447, doi:https://doi.org/10.1016/j.envint.2021.106447 (2021).

36. Croft, D. P. et al. Associations between ambient wood smoke and other particulate pollutants and biomarkers of systemic inflammation, coagulation and thrombosis in cardiac patients. Environmental Research 154, 352-361, doi:https://doi.org/10.1016/j.envres.2017.01.027 (2017).

37. Orach, J., Rider, C. F. \& Carlsten, C. Concentration-dependent health effects of air pollution in controlled human exposures. Environment International 150, 106424, doi:https://doi.org/10.1016/j.envint.2021.106424 (2021).

38. Schwartz, C., Bølling, A. K. \& Carlsten, C. Controlled human exposures to wood smoke: a synthesis of the evidence. Particle and Fibre Toxicology 17, 49, doi:10.1186/s12989-020-00375-x (2020).

39. Evans, K. A. et al. Triggering of ST-elevation myocardial infarction by ambient wood smoke and other particulate and gaseous pollutants. J Expo Sci Environ Epidemio/ 27, 198-206, doi:10.1038/jes.2016.15 (2017).

40. Hime, N. J., Marks, G. B. \& Cowie, C. T. A Comparison of the Health Effects of Ambient Particulate Matter Air Pollution from Five Emission Sources. Int J Environ Res Public Health 15, 1206 (2018).

41. Guercio, V. et al. Exposure to indoor and outdoor air pollution from solid fuel combustion and respiratory outcomes in children in developed countries: a systematic review and meta-analysis. Science of The Total Environment 755, 142187, doi:https://doi.org/10.1016/j.scitotenv.2020.142187 (2021).

42. Rokoff, L. B. et al. Wood Stove Pollution in the Developed World: A Case to Raise Awareness Among Pediatricians. Curr Probl Pediatr Adolesc Health Care 47, 123-141, doi:10.1016/j.cppeds.2017.04.001 (2017).

43. European Union. Directive 2008/50/EC of the European Parliament and of the Council of 21 May 2008 on ambient air quality and cleaner air for Europe. Official Journal of the European Union 1, 144 (2008).

44. Malmqvist, E. et al. Fetal growth and air pollution-a study on ultrasound and birth measures. Environmental research 152, 73-80 (2017). 
45. Rittner, R., Gustafsson, S., Spanne, M. \& Malmqvist, E. Particle concentrations, dispersion modelling and evaluation in southern Sweden. SN Applied Sciences 2, 1013, doi:10.1007/s42452-020-2769-1 (2020).

46. Gustafsson, S. Uppbyggnad och validering av emisssionsdatabas avseende luftföroreningar för Skåne med basår 2001 Licentiate degree thesis, Lund University, (2007).

47. Rexeis, M., Hausberger, S., Kühlwein, J. \& Luz, R. Update of Emission Factors for EURO 5 and EURO 6 vehicles for the HBEFA Version 3.2. Report No. I-31/2013, 74 (Institute for Internal Combustion Engines and Thermodynamics; Graz University of Technology Graz, Austria, 2013).

48. Windmark, F., Jakobsson, M., Segersson, D. \& Andersson, C. Underlag till uppskattning av marginalkostnader för svensk sjöfart. Modellering av ozon, sekundära partiklar och deposition av svavel och kväve. Rapport(2016).

49. Lord, C. et al. The Autism Diagnostic Observation Schedule-Generic: A Standard Measure of Social and Communication Deficits Associated with the Spectrum of Autism. Journal of autism and developmental disorders 30, 205-223, doi:10.1023/A:1005592401947 (2000).

50. Lord, C., Rutter, M. \& Le Couteur, A. Autism Diagnostic Interview-Revised: A revised version of a diagnostic interview for caregivers of individuals with possible pervasive developmental disorders. Journal of autism and developmental disorders 24, 659-685, doi:10.1007/BF02172145 (1994).

51. Haglund, N. G. S. \& Källén, K. B. M. Risk factors for autism and Asperger syndrome:Perinatal factors and migration. Autism : the international journal of research and practice 15, 163-183, doi:10.1177/1362361309353614 (2011).

52. Statistiska centralbyrån. Inkomster och inkomstfördelning (ed Enheten för statistik om befolkning och ekonomisk välfärd) 9 (SCB Stockholm, Sweden, 2021).

53. Rittner, R., Flanagan, E., Oudin, A. \& Malmqvist, E. Health Impacts from Ambient Particle Exposure in Southern Sweden. International journal of environmental research and public health 17, 5064 (2020).

54. Becerra, T. A., Wilhelm, M., Olsen, J., Cockburn, M. \& Ritz, B. Ambient Air Pollution and Autism in Los Angeles County, California. Environmental Health Perspectives 121, 380-386, doi:doi:10.1289/ehp.1205827 (2013).

55. Volk, H. E., Lurmann, F., Penfold, B., Hertz-Picciotto, I. \& McConnell, R. Traffic-Related Air Pollution, Particulate Matter, and Autism. JAMA psychiatry 70, 71-77, doi:10.1001/jamapsychiatry.2013.266 (2013).

56. Gong, T. et al. Exposure to Air Pollution From Traffic and Neurodevelopmental Disorders in Swedish Twins. Twin Research and Human Genetics 17, 553-562, doi:10.1017/thg.2014.58 (2014).

57. Gong, T. et al. Perinatal Exposure to Traffic-Related Air Pollution and Autism Spectrum Disorders. Environmental Health Perspectives 125, 119-126, doi:doi:10.1289/EHP118 (2017).

58. Guxens, M. et al. Air Pollution Exposure during Pregnancy and Childhood Autistic Traits in Four European Population-Based Cohort Studies: The ESCAPE Project. Environmental Health Perspectives 124, 133-140, doi:doi:10.1289/ehp.1408483 (2016). 
59. Ritz, B. et al. Air pollution and autism in Denmark. Environmental Epidemiology 2, e028, doi:10.1097/ee9.0000000000000028 (2018).

60. Erlandsson, L. et al. Exposure to wood smoke particles leads to inflammation, disrupted proliferation and damage to cellular structures in a human first trimester trophoblast cell line. Environmental Pollution 264, 114790, doi:https://doi.org/10.1016/j.envpol.2020.114790 (2020).

61. Assibey-Mensah, V. et al. Wintertime Wood Smoke, Traffic Particle Pollution, and Preeclampsia. Hypertension 75, 851-858, doi:doi:10.1161/HYPERTENSIONAHA.119.13139 (2020).

62. Boman, B. C., Forsberg, A. B. \& Järvholm, B. G. Adverse health effects from ambient air pollution in relation to residential wood combustion in modern society. Scandinavian Journal of Work, Environment \& Health, 251-260, doi:10.5271/sjweh.729 (2003).

63. Andersen, Z. J., Wahlin, P., Raaschou-Nielsen, O., Scheike, T. \& Loft, S. Ambient particle source apportionment and daily hospital admissions among children and elderly in Copenhagen. $J$ Expo Sci Environ Epidemio/ 17, 625-636, doi:10.1038/sj.jes.7500546 (2007).

64. Madsen, C. et al. The short-term effect of 24-h average and peak air pollution on mortality in Oslo, Norway. European Journal of Epidemiology 27, 717-727, doi:10.1007/s10654-012-9719-1 (2012).

65. Olsen, Y. et al. Emissions and source allocation of carbonaceous air pollutants from wood stoves in developed countries: A review. Atmospheric Pollution Research 11, 234-251, doi:https://doi.org/10.1016/j.apr.2019.10.007 (2020).

66. Furborg, J., Nilsson, B., Schüßler, I., Hermansson, S. \& Rönnbäck, M. Vedkaminen år 2020 - en del av boendemiljön. Report No. 02845172 (ISSN), 59 ( SP Technical Research Institute of Sweden, Borås, Sweden, 2016).

67. Oudin, A., Gustafsson, S., Rittner, R., Stroh, E. \& Malmqvist, E. På väg mot friskare luft i Skåne? , 24 (Malmö Miljöförvaltning; Lunds universitet, Lund, Sweden, 2018).

68. Szidat, S. et al. Fossil and non-fossil sources of organic carbon (OC) and elemental carbon (EC) in Göteborg, Sweden. Atmos. Chem. Phys. 9, 1521-1535, doi:10.5194/acp-9-1521-2009 (2009).

69. European Commission. European vehicle emission standards - Euro 7 for cars, vans, lorries and buses, <https://ec.europa.eu/info/law/better-regulation/have-your-say/initiatives/12313Development-of-Euro-7-emission-standards-for-cars-vans-lorries-and-buses> (2020).

70. Zhang, J., Wang, Z. \& Zhao, H. The Impact of Consumer Subsidy on Green Technology Innovations for Vehicles and Environmental Impact. Int J Environ Res Public Health 17, 7518 (2020).

71. Wang, L. et al. Air Quality Strategies on Public Health and Health Equity in Europe-A Systematic Review. Int J Environ Res Public Health 13, 1196 (2016).

72. Müller, J. \& Le Petit, Y. Low-Emission Zones are a success - but they must now move to zero-emission mobility 14 (Transport \& Environment, 2019).

73. Brooks, J. H. M., Tingay, R. \& Varney, J. Social distancing and COVID-19: an unprecedented active transport public health opportunity. British Journal of Sports Medicine 55, 411-412, doi:10.1136/bjsports-2020-102856 (2021). 
74. Barbarossa, L. The Post Pandemic City: Challenges and Opportunities for a Non-Motorized Urban Environment. An Overview of Italian Cases. Sustainability 12, 7172 (2020).

75. Viana, M. et al. Air quality mitigation in European cities: Status and challenges ahead. Environment Internationa/ 143, 105907, doi:https://doi.org/10.1016/j.envint.2020.105907 (2020).

76. Karlsson, B. S. A., Håkansson, M., Sjöblom, J. \& Ström, H. Light my fire but don't choke on the smoke: Wellbeing and pollution from fireplace use in Sweden. Energy Res Soc Sci 69, 101696, doi:https://doi.org/10.1016/j.erss.2020.101696 (2020).

77. Chafe, Z. et al. Residential heating with wood and coal: Health impacts and policy options in Europe and North America. 58 (World Health Organization, Copenhagen, Denmark, 2015).

78. Yap, P.-S. \& Garcia, C. Effectiveness of Residential Wood-Burning Regulation on Decreasing Particulate Matter Levels and Hospitalizations in the San Joaquin Valley Air Basin. Am J Public Health 105, 772-778, doi:10.2105/ajph.2014.302360 (2015).

79. Arvidsson, O., Gillberg, C., Lichtenstein, P. \& Lundström, S. Secular changes in the symptom level of clinically diagnosed autism. Journal of Child Psychology and Psychiatry 59, 744-751, doi:https://doi.org/10.1111/jcpp.12864 (2018).

80. Turner, M. C. et al. Long-Term Ozone Exposure and Mortality in a Large Prospective Study. American Journal of Respiratory and Critical Care Medicine 193, 1134-1142, doi:10.1164/rccm.201508$16330 \mathrm{C}$ (2016).

81. Lichtenstein, P., Carlström, E., Råstam, M., Gillberg, C. \& Anckarsäter, H. The Genetics of Autism Spectrum Disorders and Related Neuropsychiatric Disorders in Childhood. American Journal of Psychiatry 167, 1357-1363, doi:10.1176/appi.ajp.2010.10020223 (2010).

82. Flanagan, E., Stroh, E., Oudin, A. \& Malmqvist, E. Connecting Air Pollution Exposure to Socioeconomic Status: A Cross-Sectional Study on Environmental Injustice among Pregnant Women in Scania, Sweden. Int J Environ Res Public Health 16, 5116 (2019).

\section{Figures}




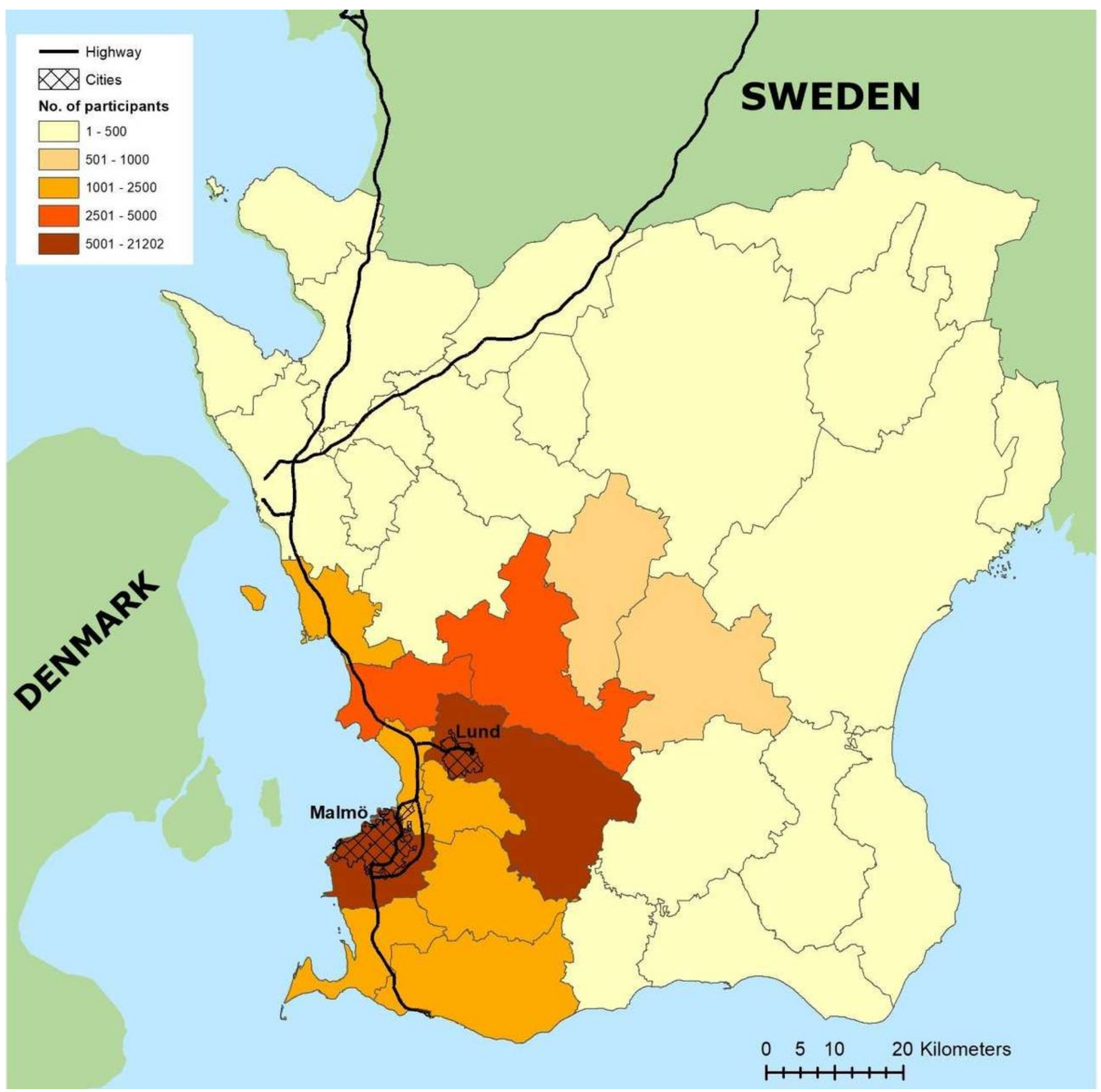

Figure 1

Distribution of the study population of the Maternal Air Pollution in Southern Sweden (MAPSS) cohort, 1999-2009. Map by Emilie Stroh. 


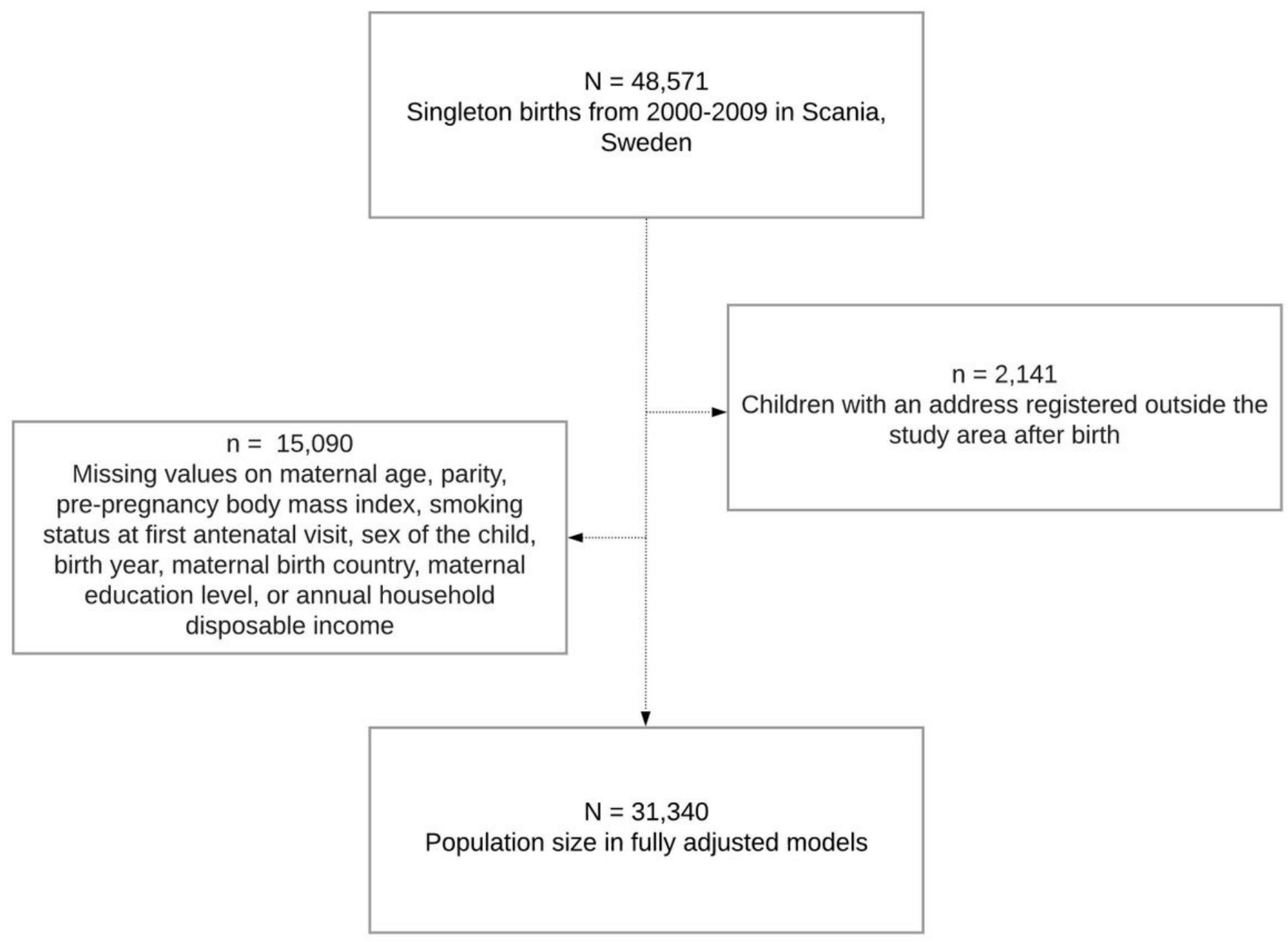

Figure 2

Flowchart of the study population by exclusion of mother/child pairs falling outside the study area and by missing data.

\section{Supplementary Files}

This is a list of supplementary files associated with this preprint. Click to download.

- Flanaganetal.SupplementaryInformation.pdf 